\title{
Rs145204276 polymorphism of GAS5 is associated with renal fibrosis via miR-21/SMAD/TGF- $\beta 1$ signaling pathway
}

\author{
Min Liu ${ }^{1}$, Guang-Chun Wang ${ }^{1}$, Yuan Feng ${ }^{2}$, Jian-Ping Che ${ }^{1}$, Hai-Min Zhang ${ }^{1}$, Yang \\ Yan ${ }^{1}$, Jian-Hua Huang ${ }^{1}$, Xu-Dong Yao ${ }^{1}$ and Jun-Hua Zheng ${ }^{1}$ \\ ${ }^{1}$ Department of Urology, Shanghai Tenth People's Hospital, Tongji University, Shanghai, 200072, China \\ ${ }^{2}$ Department of Nephrology, Nanjing University Affiliated Drum Tower Hospital, Nanjing, Jiangsu, 210093, China \\ Correspondence to: Xu-Dong Yao, email: fibrosis744@126.com \\ Jun-Hua Zheng, email: junhuazheng006@163.com
}

Keywords: renal fibrosis, GAS5, rs145204276, miR-21, SMAD/TGFB1

Received: January 02, $2017 \quad$ Accepted: April 19, $2017 \quad$ Published: January 11, 2018

Copyright: Liu et al. This is an open-access article distributed under the terms of the Creative Commons Attribution License 3.0 (CC BY 3.0), which permits unrestricted use, distribution, and reproduction in any medium, provided the original author and source are credited.

\section{ABSTRACT}

Background: Growth arrest-specific 5 (GAS5), a long non-coding RNA (IncRNA), has been identified as a key gene expression regulator in multiple cancers. The polymorphism of the promoter region of GAS5 promotes the transcription level of GAS5, and then supervises its downstream signal pathway. This study was designed to investigate the mechanism between GAS5 and renal fibrosis.

Results: rs145204276 polymorphism (INS or DEL allele) located in promoter of GAS5. The DEL/DEL of GAS5 promotes the transcription of GAS5 and lower Histological score of kidneys disease samples. QRTPCR of clinical sample show that the DEL/DEL genotype group displayed higher levels of GAS5 and SMAD7 mRNA, and lower levels of miR-21 and TGFB1 protein than INS/INS group. Further mechanism study show that GAS5 inhibits the expression level of miR-21 and then the miR-21/SMAD7/SMAD3 feedback loop. Our further study confirmed that the expression of GAS5 supervised the expression of miR21, SMAD7 and TGFB1. Meanwhile, SMAD7 is a directly target gene of miR-21.

Materials and Methods: Bioinformatics analysis and luciferase assay were employed to investigate the role of rs145204276 polymorphism in gene expression regulation. Quantitative real-time Polymerase Chain Reaction (qRT-PCR) and westernblot were employed to study the expression level. Hematoxylin-eosin staining was performed to explore role of rs 145204276 polymorphism of GAS5 in renal fibrosis.

Conclusions: our study shows that GAS5 (IncRNAs) supervises SMAD/TGF- $\beta 1$ -mediated renal fibrosis via miR-21. In addition, rs145204276 polymorphism (INS or DEL allele) of GAS5 contributes to renal fibrosis via transcription of the GAS5, and then serves as a potential therapeutic target for renal fibrosis.

\section{INTRODUCTION}

The renal tubule interstitial fibrosis (also called renal fibrosis) leads to reduced renal function [1]. For all progressive chronic kidney diseases, renal fibrosis causes the end-stage renal disease in spite of the underlying etiology. The excess accumulation of extracellular matrix and the proliferation of myofibroblasts are two main characterizations of renal fibrosis [2]. TGF- $\beta_{1}$-SMAD signaling pathway plays a key role in renal fibrosis [3]. Receptor-activated SMADs (SMAD2 and SMAD3) combine with SMAD4 to form a complex. Though moving into the nucleus and binding to DNA elements, SMADs complex promotes the transcription of various genes and involves in the renal fibrosis [3]. TGF- $\beta_{1}$-SMAD signaling is critical in the macrophage-mediated anti-GBM GN and T-cell [4]. The dysregulation of lncRNAs also relates to the TGF- $\beta_{1-}$ SMAD3 signaling pathway dysregulation, which might contribute to the renal inflammation and/or fibrosis development [5]. Comparing with mRNA, the non-coding RNAs (ncRNAs) are these RNAs which will not transcribe into proteins except tRNA and rRNA. However, these ncRNAs suppress genes' transcription and translation [6]. The well-investigated ncRNAs are micro RNA (miRNA) 
and long non-coding RNA (lncRNA). lncRNAs have been identified as the key gene expression regulators in cancer [7]. The lncRNAs are served as RNA molecules, which are longer than 200 nucleotides that are not transcribed into proteins. Recently, the roles of microRNAs in pathologic conditions, such as the renal fibrosis, where differences in the expression of mainly miR-192, miR-200, miR-21 and miR-29 have been investigated [8]. The MMP-9/TIMP1 in diabetic nephropathy is related with miR-21 in renal fibrosis, which is confirmed that MMP-9 is a target gene of miR-21 $[9,10]$. In the study of renal injury in type 2 diabetes, Zhong et al. also found the similar correlation. They found that the overexpression of miR-21 or knockdown of SMAD7 ends with more severe renal fibrosis [11]. It was also demonstrated that miR-21dependent targeting PTEN and SMAD7 was associated with the renal fibrosis progression in human diabetic nephropathy [12].

GAS5 was identified from mouse NIH $3 \mathrm{~T} 3$ cells [13]. GAS5 was introduced with stress, such as the cellcell contact inhibition and serum starvation. The human GAS5 has been shown to bind with the putative miR21-binding site at exon 4 of GAG5 and thus become a direct target of miR-21. The negative relationship between miR-21 and GAS5 has been detected in clinical breast cancer specimens [14]. One variant (rs145204276 polymorphism), located in the promoter region of GAS5, could alter the transcription ability of the promoter [15]. GAS5 functions as a sponge of miR-21, which inhibits SMAD7 expression, and SMAD7 competitively inhibits another family member of SMAD3 [16, 17]. Interestingly, SMAD3 promotes the transcription of the promoter of miR-21, and then forming a positive feedback loop (miR21/SMAD7/SMAD3), which is under control of GAS5 [16]. The presence of DEL/DEL genotype breaks the physiological hemostasis, by elevating the expression of GAS5, and thereby reducing the expression of miR-21, SMAD3. SMAD3 and its downstream effector, TGFB1, were key regulators of many pathological processes in the development of renal fibrosis; therefore, the presence of DEL/DEL genotype may reduce the risk of renal fibrosis. The above hypothesis was described in Figure 1.

\section{RESULTS}

\section{Characteristics of the participants}

The demographic and clinicopathological characteristics of the 471 renal fibrosis patients and 423 healthy controls, including gender, age, serum creatinine, protein urine and creatinine urine are shown in Table 1. The patients with and without renal fibrosis appeared to be sufficiently matched regarding gender $(P=0.575)$ and age $(P=0.8125)$ between two groups. However, serum creatinine, protein urine and creatinine urine were significantly different in patients with renal fibrosis than those without renal fibrosis. Therefore, these variables were included in the multivariate logistic regression analysis to evaluate the potential effects on the association between GAS5 rs145204276 polymorphism and the risk of renal fibrosis. We next performed logistic regression test, and found that rs 145204276 polymorphism is significantly associated with the risk of renal fibrosis (95\% CI was (0.21-0.71), OR was $0.38, P<0.01)$.

\section{GAS5 is associated with renal fibrosis}

Rs145204276 polymorphism, located in the promoter region of GAS5, could alter the transcription of GAS5. As a sponge of miR-21, GAS5 inhibits SMAD7 expression and promotes the expression of SMAD3. Interestingly, SMAD3 promotes the transcription of miR-21. Thus, miR-21/SMAD7/SMAD3 is a feedback loop, which is supervised by GAS5. The presence of DEL/ DEL genotype promotes the expression level of GAS5, and then activates its downstream genes, such as miR-21, SMAD3 and TGFB1, which influences the physiological hemostasis. The hypothesis was described in Figure 1.

\section{Rs145204276 (DEL/DEL) promotes transcription activity of GAS5}

To characterize the effect of rs145204276 polymorphism on the expression of GAS5, we constructed the vectors containing either INS allele or DEL allele upstream of a firefly luciferase reporter gene (Figure 2A). Luciferase activity assay show that rs145204276 INS/ INS promotes the transcription activity. In addition, rs145204276 DEL/DEL dramatically promotes the transcription activity of GAS5 (Figure 2B). this results suggested that the transcription activity of GAS5 promoter with DEL/DEL genotype was substantially enhanced comparing with rs 145204276 INS/INS genotype.

Furthermore, SMAD3 promoted the transcription of miR-21 in certain cell type. To test if SMAD3 promotes the expression of miR-21 in the renal tubule cells, we generated luciferase constructs with or without the promoter of miR-21 and transfected the constructs with or without SMAD3 (Figure 2C). SMAD3 significantly increases transcription of miR-21 in comparison with the empty vector. In addition, co-transfection of SMAD3 with miR-21 promoter promotes the transcription ability of miR-21 promoter (Figure 2D).

\section{SMAD7 is a direct target gene of miR-21}

SMAD7, IL-1B, STAT3 and TGF- $\beta 1$ were identified as the candidate genes with the "seed sequence" of miR-2 1 by exploring the online miRNA database. To validate these genes, we generated vectors containing wild-type or mutant 3'UTR of candidate genes: SMAD7 (Figure 3A), IL-1B (Figure 3B), STAT3 (Figure 3C) 


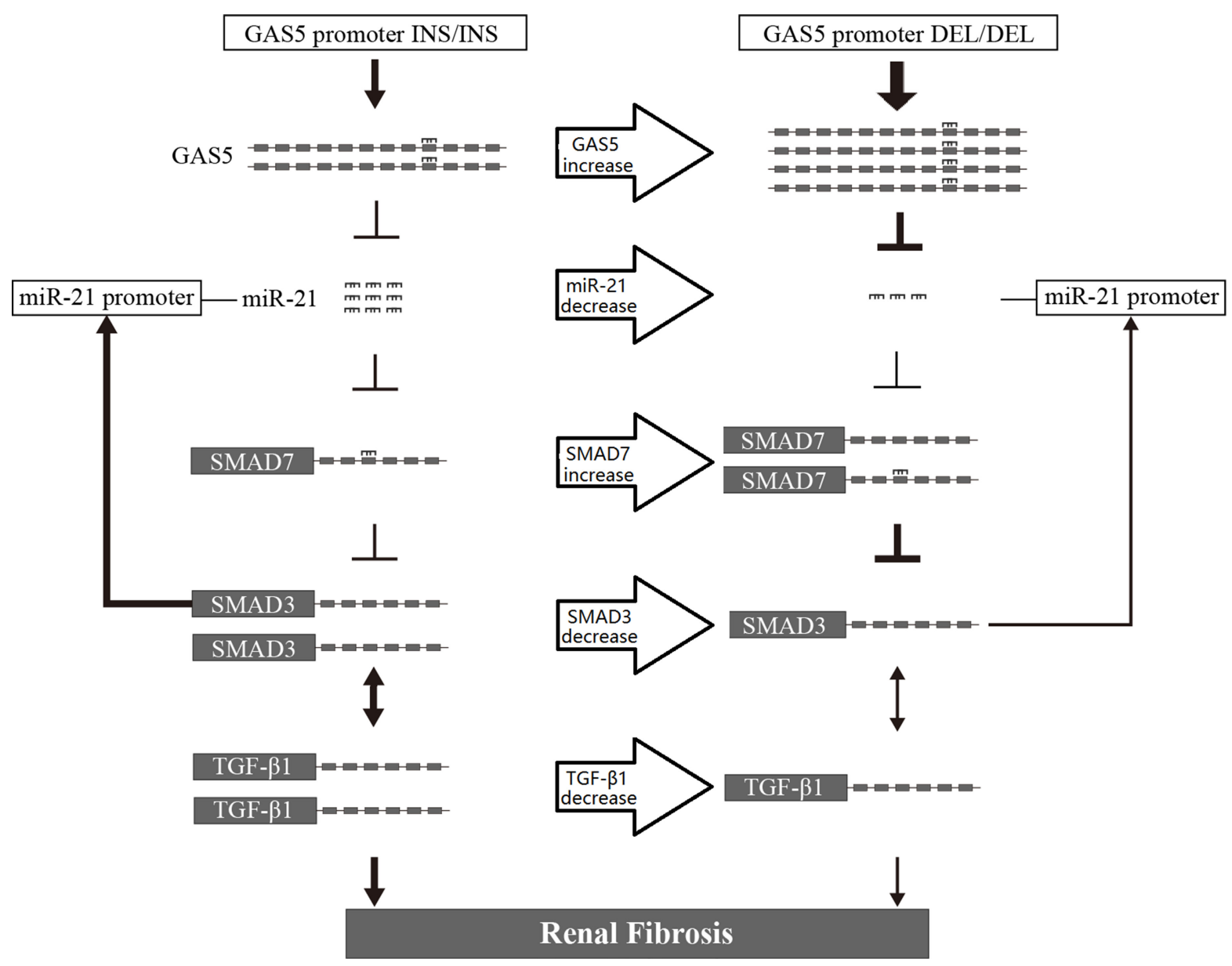

Figure 1: The expression of GAS5 is associated with renal fibrosis.
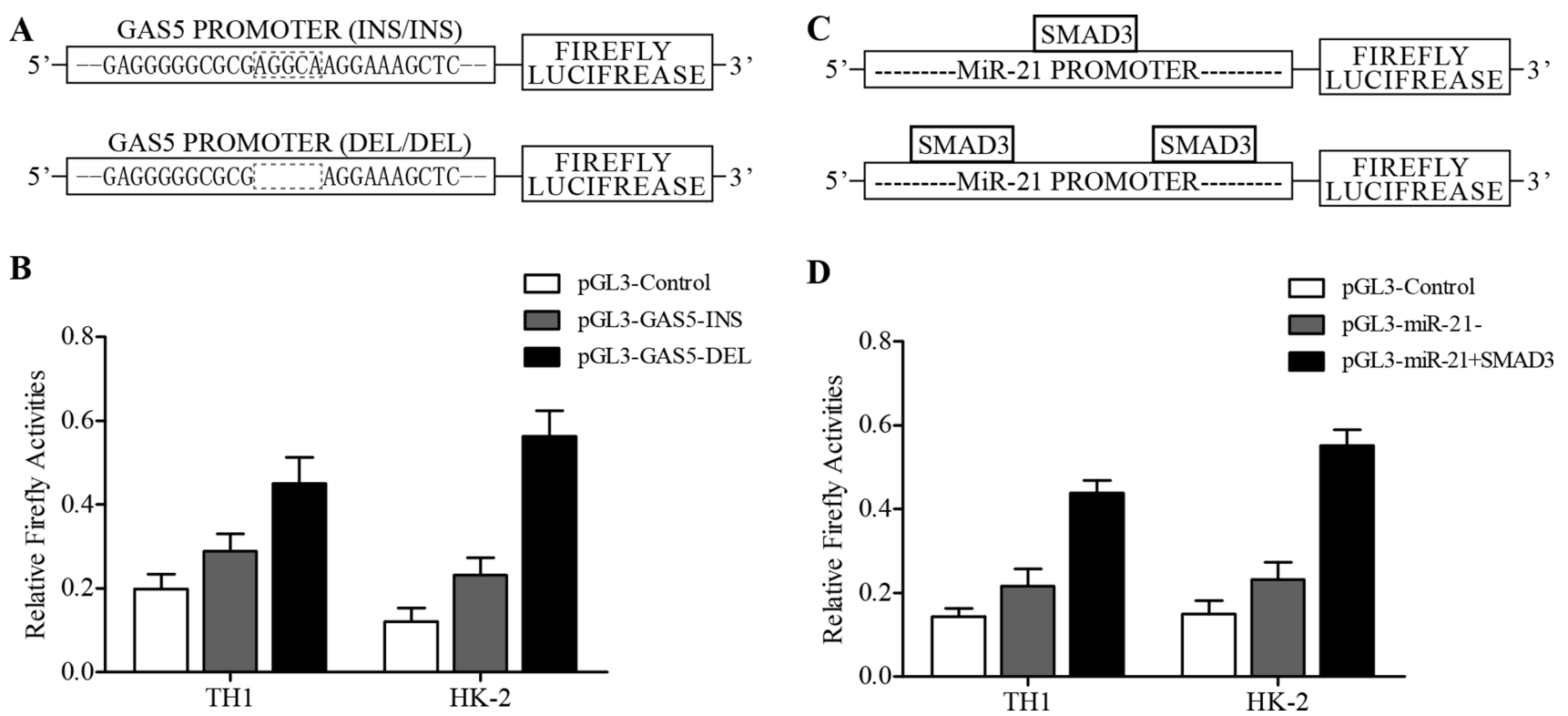

Figure 2: (A) Rs145204276 polymorphism located on GAS5 promoter, and inserted into upstream of firefly luciferase reporter gene. (B) Rs145204276 insert or deletion allele evidently increased luciferase activity of GAS5, and such effect of deletion allele was much stronger. (C) There is a binding site of SMAD3 on promoter of miR-21. (D) Luciferase assayed exhibited that SMAD3 apparently increased transcription activity of miR-21 promoter. 
Table 1: Demographic and laboratory data characteristics of groups

\begin{tabular}{lccc}
\hline & Case group $(\boldsymbol{n}=\mathbf{4 7 1})$ & Control group $(\boldsymbol{n}=\mathbf{4 2 3})$ & P value \\
\hline Gender: & & & \\
Female, $n(\%)$ & $417(88.7)$ & $378(91.5)$ & 0.573 \\
Male, $n(\%)$ & $54(11.3)$ & $45(8.5)$ & 0.8125 \\
Age (years) & $28.4 \pm 8.2$ & $31.4 \pm 6.2$ & $<0.01$ \\
Serum Creatinine (mg/d) & $2.26 \pm 0.46$ & $0.53 \pm 0.12$ & $<0.001$ \\
Protein Urine (mg/dl) & $785.32 \pm 428.2$ & $46.25 \pm 33.4$ & $<0.001$ \\
Creatinine Urine (mg/dl) & $74.56 \pm 58.1$ & $142.48 \pm 74.5$ & \\
Genotype rs145204276 & & & \\
INS/INS, $n(\%)$ & $198(46.8)$ & OR 0.38 \\
INS/DEL, $n(\%)$ & $226(48.2)$ & $190(44.9)$ & $95 \%$ CI $(021,0.71)$ \\
Carrier of INS (\%) & $229(48.4)$ & $388(91.7)$ & $<0.01$ \\
DEL/DEL, $n(\%)$ & $455(96.6)$ & $35(8.3)$ & \\
\hline
\end{tabular}

and TGF- $\beta 1$ (Figure 3D). MiR-21 inhibits the luciferase activity of the SMAD7-3'UTR. Meanwhile, miR-21 does not inhibit the luciferase activity of SMAD7-3'UTR-mut. On the contrary, miR-21 does not supress IL-1B, STAT3 orTGFB1. Therefore, miR-21 directly suppresses the expression of SMAD7, but not IL-1B, STAT3 and TGFBI.

\section{MiR-21 inhibits the expression of SMAD7}

To investigate if miR-21 suppress the expression of SMA7, IL-1B, STAT3 and TGF- $\beta 1$, we transfected miR-21 mimics or anti-miR-21 into cells and the real-time PCR and western-blot were utilized to identify the effect of change in miR-21 on expression of candidate target genes including SMAD7, IL-1B, STAT3 and TGF- $\beta 1$, and the expression of those candidate target genes were determined and compared between cells transfected with miR-21 mimic and negative control in HK-2 cells. As shown in Figure 4, only SMAD7 mRNA (Figure 4A) and protein (Figure 4B) levels were apparently decreased in miR-21 treatment group, while mRNA (Figure 4A) and protein (Figure 4B) levels of IL-1B, STAT3 and TGF- $\beta 1$ showed no obvious difference between miR-21 treatment and $\mathrm{NC}$ groups. In line with this, downregulation of miR21 by the transfection with anti-miR-21 could upregulate the expression of SMAD7 protein (Figure 4C) and mRNA (Figure 4D) in the same cells, while the underexpression of miR-21 had minimal effect on the expression of protein (Figure 4C) and mRNA (Figure 4D) of IL-1B, STAT3 and TGF- $\beta 1$.

\section{Rs145204276 polymorphism mediates the expression of miR-21}

To investigate if the rs 145204276 polymorphism regulates the expression of miR-21, SMAD7 and TGF- $\beta 1$ expression, we collected kidneys tissues (INS/INS, $n=12$,
INS/DEL, $n=8$, DEL/DEL $n=5$ ), and qRTPCR and western-blot were performed to determine the expression of each gene. As shown in Figure 5, GAS5 (Figure 5A), SMAD7 (Figure 5C) were highly expressed in DEL/DEL genotype group compared with that in INS/INS or INS/ DEL group, and on the contrary miR-21 (Figure 5B) and TGF- $\beta 1$ protein (Figure 5D) were highly expressed in INS/INS or INS/DEL genotype group than that of DEL/ DEL group.

\section{Rs145204276 polymorphism is associated with renal fibrosis}

The histological slides (hematoxylin-eosin stain as shown in Figure 6A and 6B, and mason stain as shown in Figure $6 \mathrm{C}$ and $6 \mathrm{D}$ ) were collected to study the impacts of rs145204276 polymorphism on the severity of renal fibrosis. The histological score (hematoxylin-eosin stain) of Kidneys tissue with DEL/DEL genotypes show significantly decrease comparing with that of INS/INS or INS/DEL samples (Figure 6A). The representative histological slides of renal fibrosis were shown in Figure 6B. The histological score (mason stain) of Kidneys tissue with DEL/DEL genotypes show significantly decrease comparing with that of INS/INS or INS/DEL samples (Figure 6C). The representative histological slides of renal fibrosis were shown in Figure 6D. These data confirmed that rs 145204276 polymorphism DEL/DEL is associated with lower level renal fibrosis comparing with INS/INS or INS/DEL genotype.

\section{GAS5 supervises the expression of miR-21, SMAD7 and TGF- $\beta 1$}

To examine if GAS5 supervises the expression of miR-21, SMAD7, SMAD3 and TGF- $\beta 1$ in HK2 cells, GAS5 siRNA or GAS5 mimic was transfected 
into the cells. 2 days later, the expression level of miR-21, SMAD7, SMAD3 and TGF- $\beta 1$ were determined via western blot and qRT-PCR. The expression level of miR-21 was significantly decreased by the transfection of pcDNA3-GAS5 (Figure 7A). The mRNA and protein level of SMAD7 was significantly increased by the transfection of pcDNA3-GAS5 (Figure 7B). Similarly, the mRNA and protein of TGF- $\beta 1$ was significantly increased by the transfection of pcDNA3-GAS5 (Figure 7D). However, the expression of SMAD3 was not influenced by the transfection with pcDNA3-GAS5 (Figure 7C).

To further investigate the regulation role of GAS5, we transfected GAS5 siRNA into TH1cells and examine the expression miR-21, SMAD7 and TGF- $\beta 1$. The expression level of miR-21 was significantly increased by GAS5 siRNA transfection (Figure $8 \mathrm{~A}$ ). The mRNA level and protein level of SMAD7 or TGF- $\beta 1$ was significantly decreased by the GAS5 siRNA transfection (Figure 8B and 8D). The mRNA level and protein level of SMAD3 was not suppressed d by the GAS5 siRNA transfection (Figure 8C).

\section{DISCUSSION}

The expression of GAS5 is associated with multiple kinds of cancers. In addition, the loss of GAS5 is related to poor prognosis of cervical cancer, $\mathrm{HCC}$ and gastric cancer [18]. Moreover, the SNP of the promoter region
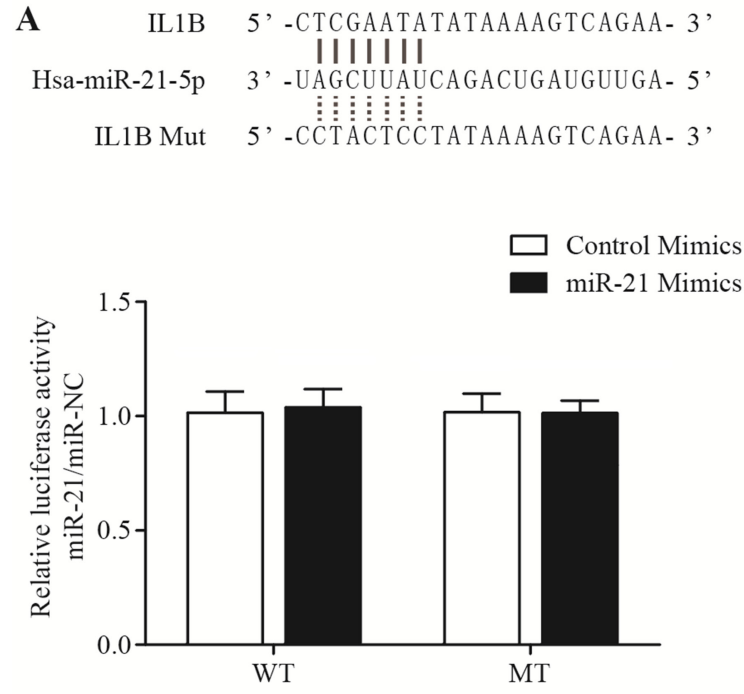

B
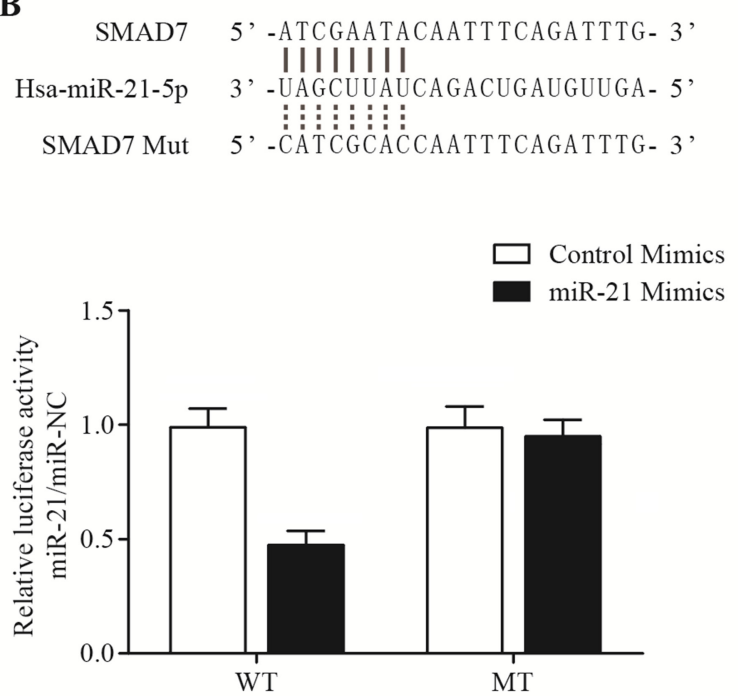

C
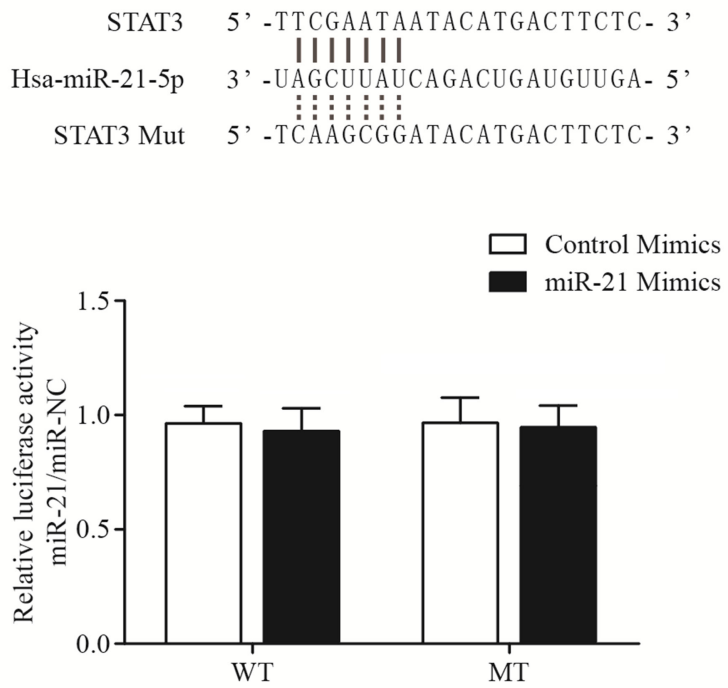

D
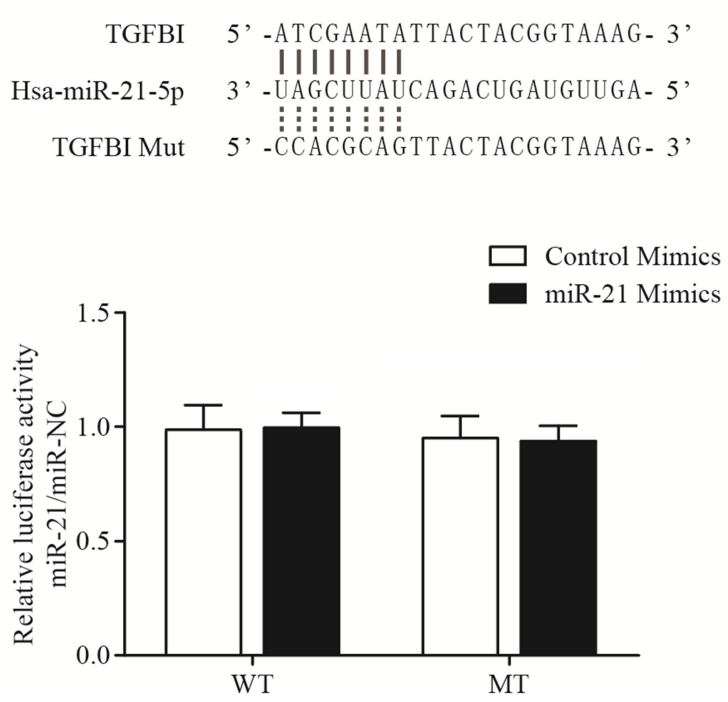

Figure 3: SMAD7 is a direct target gene of miR-21. (A) Bioinformatics predicts that IL-1B had a "seed sequences" of miR-21, and miR-21 had no effect on luciferase activity of wild-type or mutant IL-1B 3'UTR in HK2 cells. (B) Bioinformatics predicts that SMAD7 had a "seed sequences" of miR-21, and and miR-21apparently attenuated luciferase activity of wild-type SMAD7 3'UTR in HK2 cells. (C) Bioinformatics predicts that STAT3 had a "seed sequences" of miR-21, and miR-21 had no effect on luciferase activity of wild-type or mutant STAT3 3'UTR in HK2 cells. (D) Bioinformatics predicts that TGFB1 had a "seed sequences" of miR-21, and miR-21 had no effect on luciferase activity of wild-type or mutant TGFB1 3'UTR in HK2 cells. 

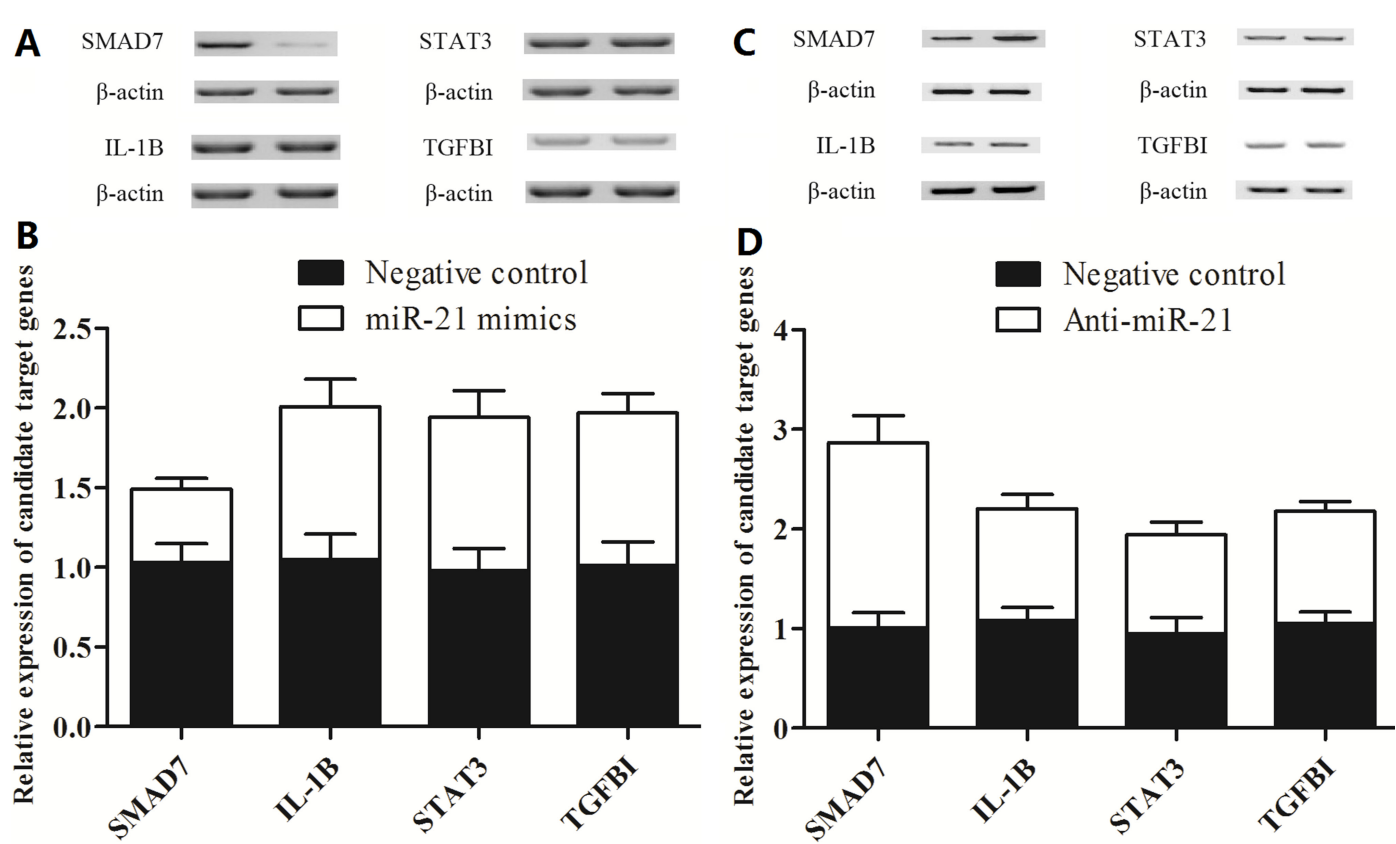

Figure 4: Real-time PCR and western-blot were performed to study the effect of miR-21 on expression of SMAD7, IL-1B, STAT3 and TGFB1 in HK-2 cells. (A) Only SMAD7 protein was significantly downregulated between miR-21 treatment and NC groups, while the expression of IL-1B, STAT3 and TGFB1 was minimally affected. (B) Only SMAD7 mRNA was significantly downregulated between miR-21 treatment and NC groups, while the expression of IL-1B, STAT3 and TGFB1 was minimally affected. (C) Only SMAD7 protein was significantly upregulated between anti-miR-21 treatment and NC groups, while the expression of IL-1B, STAT3 and TGFB1 was minimally affected. (D) Only SMAD7 mRNA was significantly upregulated between anti-miR-21 treatment and NC groups, while the expression of IL-1B, STAT3 and TGFB1 was minimally affected.
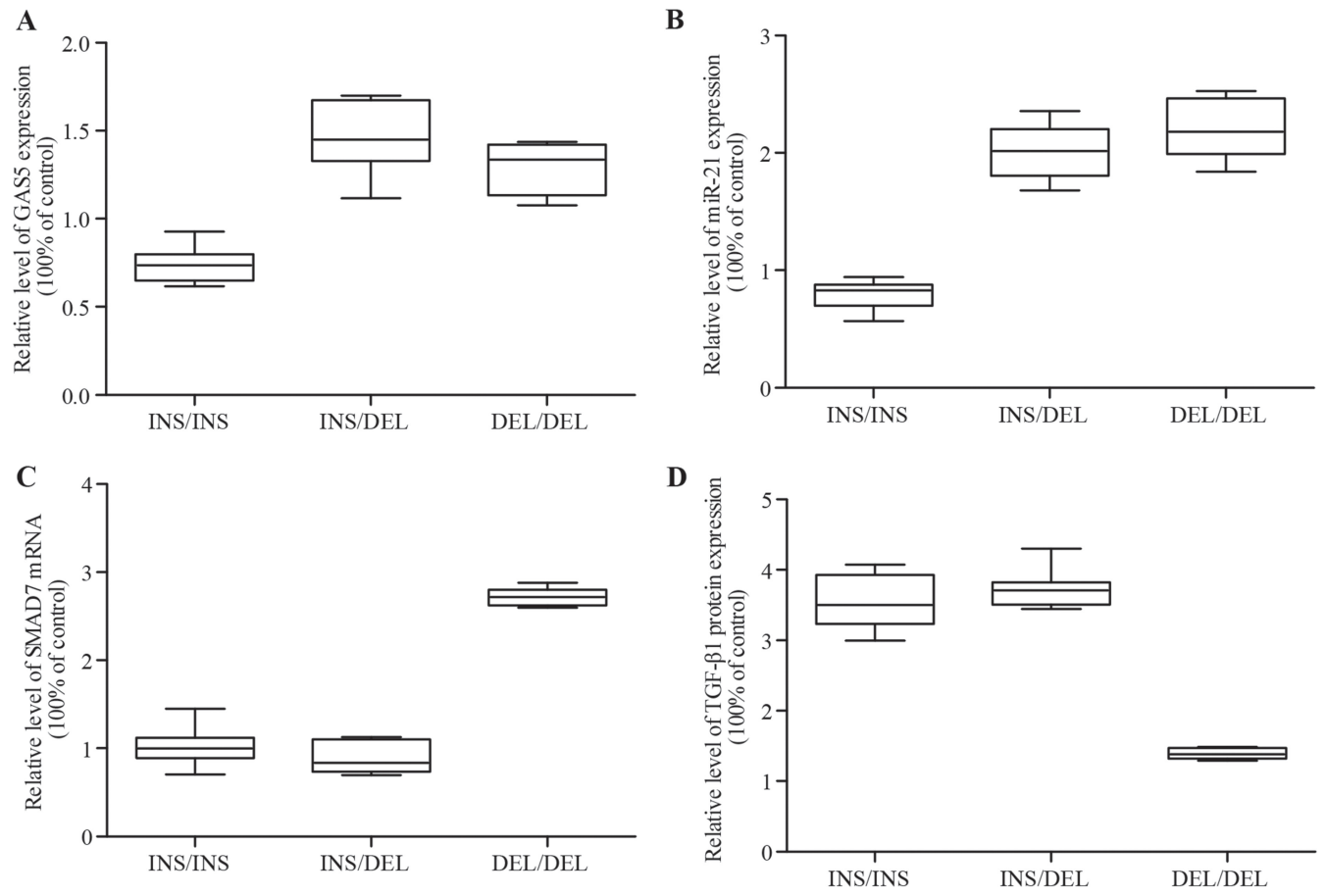

Figure 5: The rs145204276 polymorphism of GAS5 supervises the expression of miR-21, SMAD7 and TGFB1. (A) GAS5 level was much lower in tissues genotyped as DEL/DEL. (B) MiR-21 level was much higher in tissues genotyped as DEL/DEL. (C) SMAD7 mRNA level was much lower in tissues genotyped as DEL/DEL. (D) TGFB1 protein level was much higher in tissues genotyped as DEL/DEL. 
of lncRNA IRAIN can alter the methylation status of the CpGs island and thus govern the expression level of GAS5 in the breast cancer [19]. The rs 145204276 polymorphism of promoter region of GAS5 was related to susceptibility to HCC. The SNP alters a $\mathrm{CpG}$ island methylation status in the GAS5 promoter region, and then the transcriptional activity of GAS5 and finally the expression level of GAS5 [15].The promoter region of GAS5 the deletion allele of a 5-base pair indel polymorphism (rs145204276) greatly enhanced the expression of GAS5 in hepatocellular cell lines and increased the risk of hepatocellular carcinoma (HCC), and it indicated that the GAS5 played differential roles in carcinogenesis of different cancer types [15]. To examine if the rs 145204276 polymorphism influences the expression of GAS5, we conducted luciferase assay and found that that the deletion allele significantly increased luciferase activity of GAS5. Furthermore, 25 kidney tissue samples were collected from renal fibrosis patients, and we found that GAS5 and SMAD7 were highly expressed in DEL/DEL genotype group, and on the contrary miR-21 and TGFB1 protein were highly expressed in INS/ INS genotype group. Our data show that rs 145204276 polymorphism is significantly associated with the risk of renal fibrosis in a population of 894 participants consisting of 471 renal fibrosis patients and 423 controls. Recently, non-coding RNAs, including lncRNAs and miRNAs, can be served as miRNA sponges [20]. The loss of GAS5 is associated with upregulation of miR-21in breast tumor tissues, which suggested that GAS5 is negatively correlated with miR-21 [14]. A reciprocal repression of miR-21 and GAS5 has been found during OA pathogenesis. The expression of GAS5 was significantly increased by miR-21 inhibition which leads to the degradation of cartilage [21].

SMAD7 is a direct target of miR-21 in lung fibrosis. The expression of SMAD7 and miR-21 is negatively correlated [22]. In addition, the loss of miR-21 leaded to the restore of SMAD7, which was accompanied by deceased SMAD3 phosphorylation [11]. In a word, miR-21 is associated with renal fibrosis via multiple roles in cell metabolism and cell signaling pathways regulation [16]. Using computational analysis, we identified four candidate genes which might be functionally involved in the pathogenesis of renal fibrosis, and validated SMAD7 as a direct target gene of miR-21 in renal tubule cells using luciferase reporter system. And the relationship was further confirmed by the observation that the expression of SMAD7 was inhibited in the culture cell transfected with
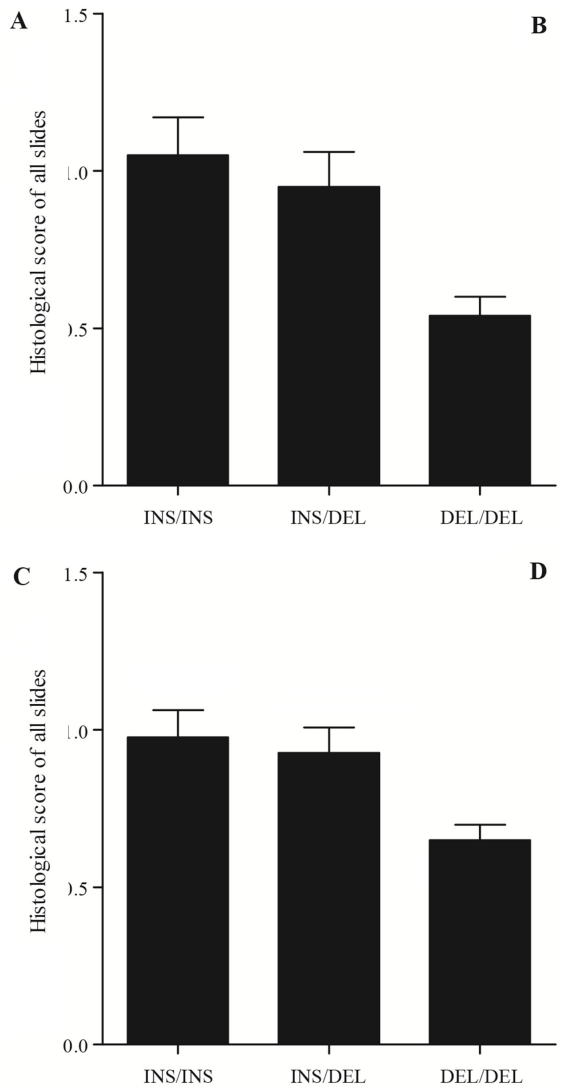

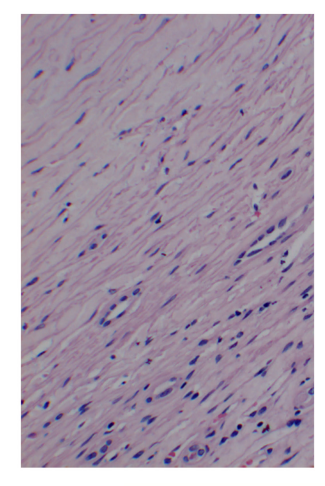

INS/INS

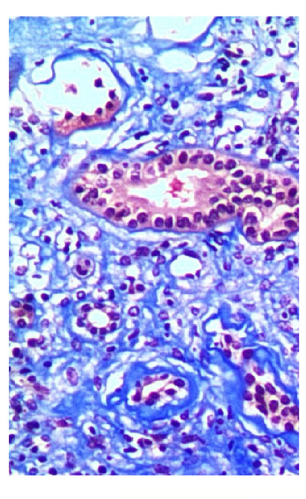

INS/INS

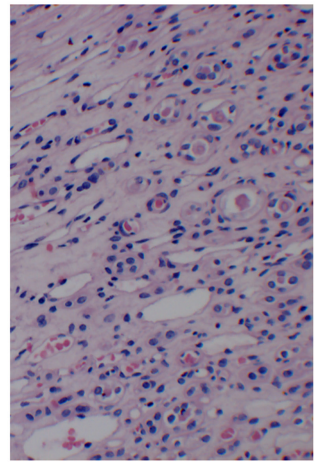

INS/DEL

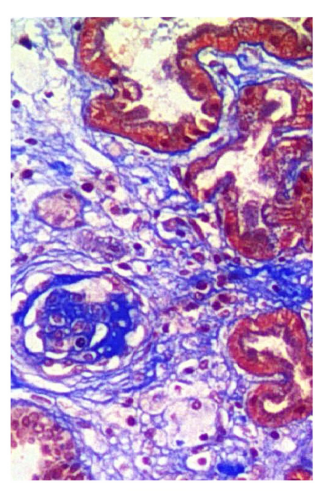

INS/DEL

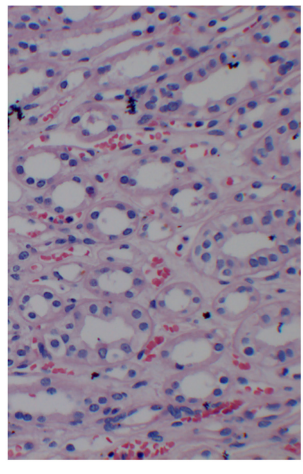

DEL/DEL

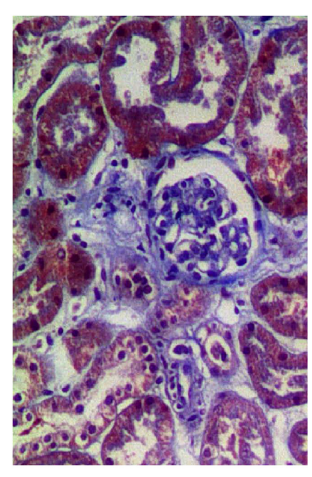

DEL/DEL

Figure 6: The rs145204276 polymorphism is associated with renal fibrosis. (A) Histological score of all slides (H\&E stain) in kidneys tissues genotyped as DEL/DEL was much lower. (B) The representative histological slide (H\&E stain) of renal fibrosis in individuals genotyped as DEL/DEL, INS/INS and INS/DEL. (C) Histological score of all slides c in kidneys tissues genotyped as DEL/ DEL was much lower. (D) The representative histological slide (H\&E stain) of renal fibrosis in individuals genotyped as DEL/DEL, INS/ INS and INS/DEL. 
miR-21 mimics compared with the control, while other candidate gene expression was comparable between the two groups.

The transforming growth factor-b1 (TGF-b1), a well-known pro-fibrogenic cytokine, activates its downstream mediators (SMAD2 and SMAD3) which are counter inhibited by an inhibitory SMAD7, and thus plays critical roles in multiple functions [23]. SMAD3 is a key transcription factor and involved in multiple fibrogenic mediators response. Loss of SMAD3 impairs immunity and result in the autoimmune disease [24]. SMAD/ TGF- $\beta 1$ signaling is critical in renal inflammation and fibrosis in some renal diseases [25, 26]. The SMAD3 activation is related to renal fibrosis and mediates the production of collagen which is induced by advanced glycated end products (AGEs), TGF- $\beta 1$, vascular smooth muscle cells, high glucose, tubular epithelial cells (TEC), and angiotensin II (AngII) in renal tubule cells [27]. The overexpression of SMAD7 inhibits the function of SMAD3 and thereby the signaling pathway of SMAD/ TGF- $\beta 1$, demonstrating that TGF- $\beta 1$ governs renal fibrosis inhibited by SMAD7, but positively by SMAD3 [27].

The SMAD2/3 canonical effectors of TGF- $\beta 1$ signaling promote pri-miR-21's post-transcriptional cleavage into pre-miR-21 by DROSHA [28]. Phosphorylated SMAD3 promoted pri-miR-21's transcriptional activation and maturation. [29]. The overexpression of SMAD3 leads to the upregulation of miR-21. On the contrary, Loss of SMAD3 is related to a considerably lower production of an accumulation of pre-miR-21 and maturation of miR-21 within the cells [30]. In renal inflammation, loss of SMAD7 enhances
A

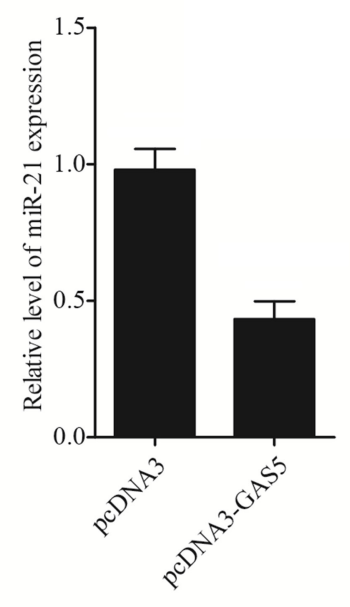

C

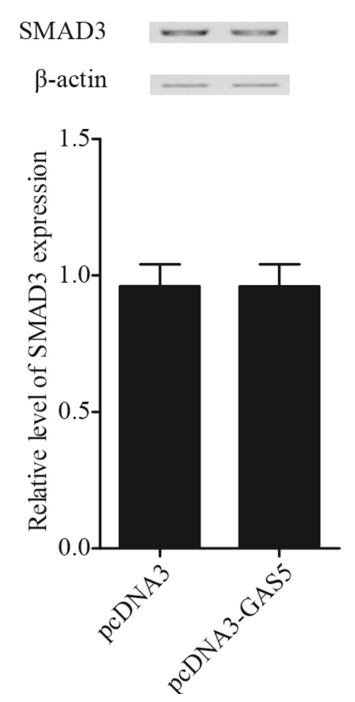

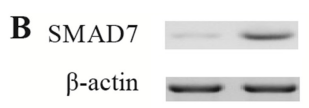

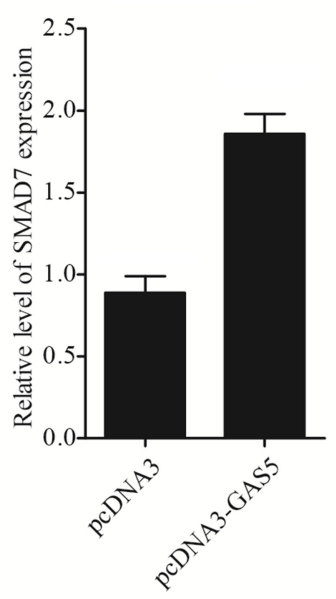

D

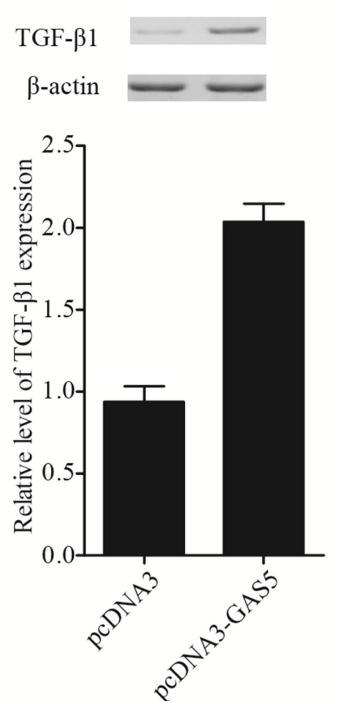

Figure 7: GAS5 mimic supervises the expression of miR-21, SMAD7 and TGFB1 in HK-2 cells. (A) MiR-21 expression was inhibited by GAS5 mimic. (B) SMAD7 mRNA and protein levels were increased by GAS5 mimic. (C) GAS5 had no effect on SMAD3 mRNA and protein expressions. (D) TGFB1 mRNA and protein levels were increased by GAS5 mimic. 
SMAD/TGF- $\beta 1$ signaling pathway. On the contrary, the overexpression of SMAD7 impairs SMAD3 signaling, and then suppresses the renal fibrosis and inflammation [31]. Loss of SMAD3 in mice inhibited renal fibrosis and inflammation in angiotensin II-induced hypertensive nephropathy and unilateral ureteral obstructive nephropathy (UUO). This finding further confirmed that SMAD3 is critical in in renal fibrosis and inflammation [32]. The role of SMAD/TGF- $\beta 1$ in renal inflammation

A

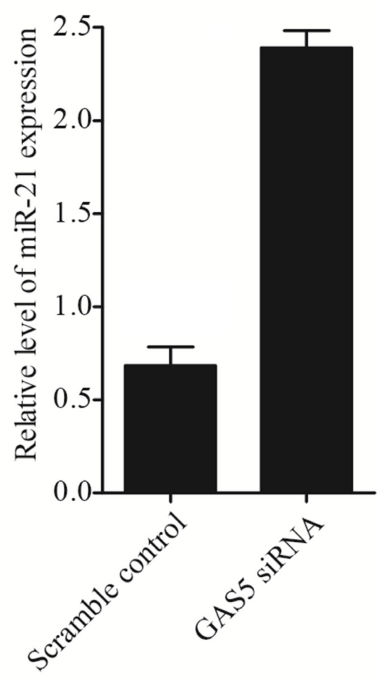

C
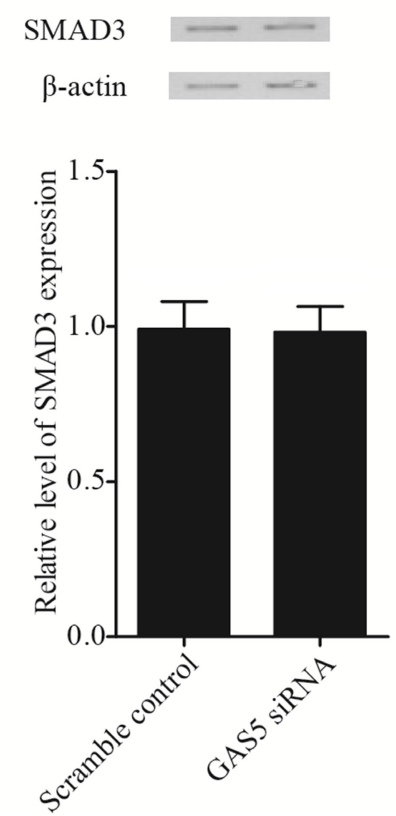

and fibrosis indicates that SMADs are better target than TGF- $\beta 1$ in the kidney disease with inflammation and/or fibrosis. Based on the above evidence, we confirmed that miR-21, SMAD3, SMAD7 form a positive feedback loop, which is supervised by GAS5. We confirmed that SMAD3 promotes the miR-21 transcription activity. In addition, the upregulation of GAS5 in the culture renal tubule cells via rs145204276 DEL allele inhibits the express level of miR-21 and promotes the level of SMAD7, and then

B

\section{SMAD7 \\ $\beta$-actin}
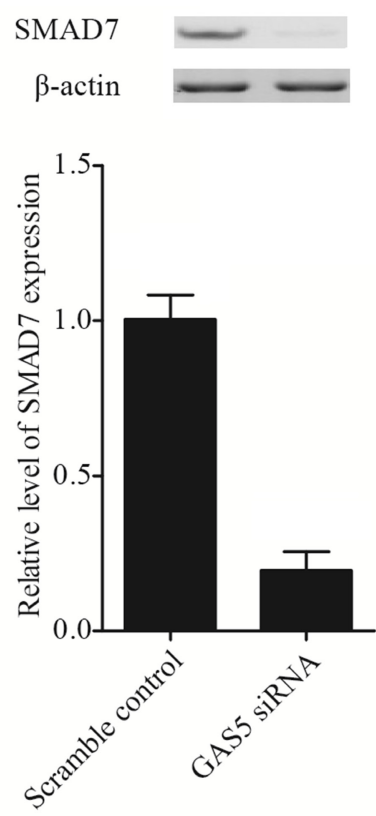

D
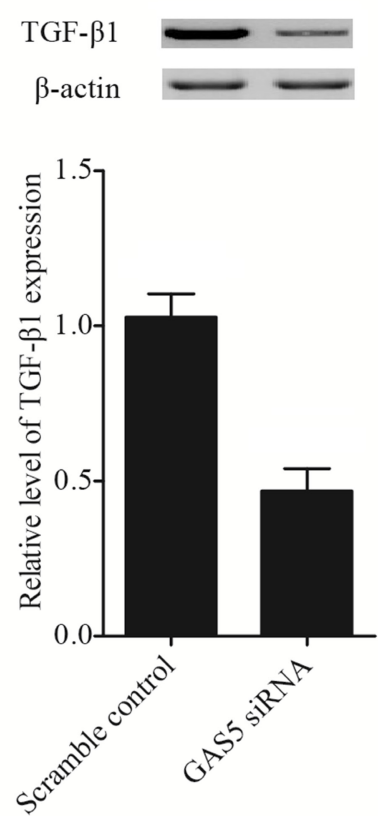

Figure 8: Loss of GAS5 by siRNA supervises the expression of miR-21, SMAD7 and TGFB1 in TH1 cells. (A) Transfecting with GAS5 siRNA promoted miR-21 expression. (B) Transfecting with GAS5 siRNA apparently inhibited SMAD7 mRNA and protein levels. (C) GAS5 siRNA had no effect on SMAD3 mRNA and protein expressions. (D) Transfecting with GAS5 siRNA apparently inhibited TGFB1 mRNA and protein levels. 
subsequently suppressed expression of SMAD3, and the TGF- $\beta 1$ signaling, resulting in less risk of renal fibrosis.

\section{MATERIALS AND METHODS}

\section{Subjects}

Human Research Ethics Committees of Shanghai Tenth People's Hospital, Tongji University (Shanghai, China) approved this research. 471 patients with renal fibrosis were recruited from Department of Urology, Shanghai Tenth People's Hospital, Tongji University (Shanghai, China) as case group, and 423 participants without renal fibrosis were enrolled as control groups. $5 \mathrm{ml}$ pf peripheral blood was collected from each participant. The demographic and clinicopathological characteristics of patients, such as age, gender, and renal function were collected and described in Table 1. Written informed consent was obtained from all patients or their first-degree relatives after explained all the potential risk. The study was conducted according to the Declaration of Helsinki. In addition, 25 kidney tissue samples were collected from 25 patients with diagnosis of renal fibrosis, which was approved by institutional review board and the kidney samples were stored at liquid nitrogen for the functional analysis such as real-time PCR, western blot and immunehistochemistry.

\section{Genotyping}

Genomic DNA was collected from tissue samples by using QIAampDNA Blood Mini Kit from Qiagen (Germany). Genotyping analysis was performed using TaqMan allele discrimination assays (Applied Bio-systems, Foster City, CA) based on the protocol from suppliers. The TaqMan Geno-typing Universal PCR Master Mix (Applied Bio-systems, Foster City, CA) automatically detected genotypes by the ratio of the two fluorophores used.

\section{MicroRNA and IncRNA RNA isolation and quantitative real-time PCR}

Trizol reagent (Invitrogen, CA, US) was utilized to extract RNA from culture cells or tissue samples based on supplier's instruction. SuperScript ${ }^{\circledR}$ IV First-Strand Synthesis System (Invitrogen, CA, USA) was utilized to reverse-transcribe total RNA to cDNA in a $50 \mu \mathrm{L}$ reaction mixture with $0.5 \mu \mathrm{g}$ of total RNA, $1 \mu \mathrm{L}$ sense primer, 1 $\mu \mathrm{L}$ anti-sense, $25 \mu \mathrm{L}$ Premix Taq and water sterilized distilled to $50 \mu \mathrm{L}$ in accordance with manufacturer's recommendation. And the thermal cycles were carried out at $95^{\circ} \mathrm{C}$ for $5 \mathrm{~min}, 94^{\circ} \mathrm{C}$ at $30 \mathrm{~s}$ for 30 cycles, $55^{\circ} \mathrm{C}$ at $50 \mathrm{~s}$, $72^{\circ} \mathrm{C}$ for $60 \mathrm{~s}$, and final $72^{\circ} \mathrm{C}$ for $5 \mathrm{~min}$. Applied Biosystems Primer Express 3.0 (Applied Biosystems, Foster city, California, USA) was utilized to design the primers. 7500 Fast Real-Time PCR System (Applied Biosystems, Foster city, California, USA) was utilized to perform real-time PCR in a $25 \mu \mathrm{L}$ with $12.5 \mu \mathrm{L}$ of $2 \times$ SYBR Fast qPCR Mix (Takara, Kyoto, Japan), $2 \mu \mathrm{L}$ cDNA, $1 \mu \mathrm{L}$ PCR reverse Primer $(10 \mu \mathrm{m}), 1 \mu \mathrm{L}$ of PCR forward Primer $(10 \mu \mathrm{m})$ and $8.5 \mu \mathrm{L}$ water without nuclease. And thermal cycles were carried out at $95^{\circ} \mathrm{C}$ for $30 \mathrm{~s}, 95^{\circ} \mathrm{C}$ at $3 \mathrm{~s}$ for 40 cycles, $60^{\circ} \mathrm{C}$ for $15 \mathrm{~s}$. The specific generation of PCR products expected was validated with the use of melting curve analysis at the end of thermal cycles, and $2^{-\Delta \Delta C t}$ method was performed to calculate the expression levels of GAS5, miR-21 and TGFBI, STAT3, SMAD7, SMAD3 mRNA using the following primer sets: GAPDH: Forward 5'-TGG TATCGTGGAAGGACTCAT-3' and reverse 5'-GTGGG TGTCGCTGTTGAAGTC-3'; miR-21: Forward 5'-GCGC GTCGTGAAGCGTTC-3' and reverse 5'-GTGCAGGGT CCGAGGT-3'; GAS5: Forward 5'-AGCTGGAAGTTGA AATGG-3' and reverse 5'-CAAGCCGACTCTCCAT ACC-3'; SMAD7: Forward 5'-GTGTTGCTGTGAATC TTACGG-3' and reverse 5'-GATGAAGATGGGGTAAC TGCT-3'; SMAD3: Forward 5'-GGATGGTCGGCTGCA GGTGTCC-3'and reverse 5'-TGTTGAAGGCAAACT CACAGAGC-3'; TGFB1: Forward 5'-GTGGACCGCAA CAACGCCATCT-3'and reverse 5'-GCAATGGGGGTT CGGGCACT-3'; STAT3: Forward 5'-TGTTGGAGCAGCA TCTTCAG-3'and reverse 5'-GAGGTTCTCCACCACC TTCA-3' Target gene expression levels were normalized to those of the control gene (GAPDH for mRNA or U6 for miRNA). Three independent tests were run.

\section{Cell culture and transfection}

RPMI 1640 medium (Gibco, Invitrogen, Carlsbad, CA, USA) contained 10\% FBS (fetal bovine serum), 100 $\mathrm{mg} / \mathrm{mL}$ streptomycin and $100 \mathrm{U} / \mathrm{mL}$ penicillin (Beyotime Biotech, Haimen, China) was utilized to maintain the TH1 and HK2 cells with $5 \% \mathrm{CO}_{2}$ at $37^{\circ} \mathrm{C}$. Full length of GAS5 or the coding domain sequence of (CDS) of SMAD3 was amplified using PCR and inserted into pcDNA3.0. GAS5 siRNA or the scramble control were purchased from Gene Pharma (Shanghai, China), and Lipofectamine ${ }^{\mathrm{TM}} 2000$ reagent (Invitrogen, CA, US) was utilized to transfect cells with pcDNA3.0, pcDNA3-GAS5, GAS5 siRNA (Cat\# 1299001, thermo fisher, ), miR-21 mimic (5'UAGCUUAUCAGACUGAUGUUGA-3'), anti-miR-21 mimic (5'-UCAACAUCAGUCUGAUAAGCUA-3') and scramble control at a final concentration of $30 \mathrm{nM}$ following recommendation provided by manufacturer. We harvested for further analysis 48 hours after transfection. All experiments were run in triplicate.

\section{Luciferase assay to test the regulatory association between miR-21 and the candidate genes}

Dual-Luciferase Reporter Assay System (Promega, Madison, WI, USA) was utilized to perform luciferase 
assay following manufacturer's instruction. The fulllength of 3'UTR of the candidate target genes such as SMAD7, STAT3, TAT3 and TGFBI were amplified and cloned into multiple restrictive sites of psiCHECK-2 vector located on downstream of the luciferase gene, DNA sequencing was performed to confirm all constructs. And before transfection, the TH1 and HK2 cells were seeded into 48-well plates, and when cells reached 80 confluences, the cells were transfected recombination constructs and psiCHECK-2 luciferase vector, and miR-21 mimic or miRNA control with the use of Lipofectamine 2000 (Invitrogen, CA, USA) following guideline by the manufacturer. 48 hours after transfection, washed the cells twice, and lysis buffer was utilized to lyse cells, and dual luciferase reporter assay system (Promega, Madison, WI, USA) with a luminometer was utilized to determine luciferase activity of firefly, and the relative firefly luciferase activity was detected normalized to luciferase activity of control. Three independents experiments were carried out.

\section{Luciferase assay to test the effect of variant and introduction of SMAD3 on the transcription of the candidate genes}

In brief, PCR was performed to amplify full promoter region (either the promoter of GAS5 and miR-21) amplified from samples derived from subjects with different genotype. The GAS5 promoter-luciferase reporter plasmids contained either rs 145204276 with INS or DEL allele, and the promoter of miR-21 is wild-type. Endonuclease (NEB, BioLabs Inc., CA, US) was utilized to digest pGL3-basic vector (Promega, MD, USA) and the amplified promoter region of GAS5 (containing DEL or INS allele) or the promoter of miR-21 was inserted into the pGL3 with a firefly reporter gene downstream of each inserted promoter. Lipofectamine 2000 (Invitrogen, Carlsbad, CA, USA) was utilized to transfect the cells with pGL3, pGL3-GAS5 promoter-INS, pGL3-GAS5 promoter-DEL, pGL3-miR-21 promoter (with or without introduction of SMAD3). 48 hours post-transfection, Dual Luciferase assay system (Promega Corp., Madison, WI, USA) was utilized to detect luciferase activity of firefly normalized to that of Renilla. Each test was carried out three times.

\section{Western blot analysis}

A modified radio-immunoprecipitation assay lysis buffer (Upstate Biotechnology, Lake Placid, NY, USA) with protease inhibitor cocktail (Sigma Aldrich, CA, USA) was utilized to lyse the culture cells or tissue samples 48 hours after transfection to detect the expression level of CANCAC1 protein in accordance with manufacturer's instruction. 4-20\% SDS gradient gels (Mini-PROTEAN TGX; Bio-Rad, Hercules, CA) were utilized to load equal amounts $(30 \mu \mathrm{g})$ of total protein, and gel electrophoresis was utilized to separate the protein, and then transferred to PVDF membranes (Immobilon-P; Millipore, Billerica, MA, USA). And then the primary antibodies anti- TGFBI, IL-1B, STAT3, SMAD7, (all primary antibodies purchased from Cell Signaling Technology, Inc., Beverly, MA, USA) and monoclonal anti- $\beta$-actin at a dilution of 1:10000 (cat. no.sc-130301; Santa Cruz Biotechnology, Santa Cruz, CA, USA) were utilized to treat the membrane at $4{ }^{\circ} \mathrm{C}$ overnight, and Horseradish peroxidase (HRP)-conjugated secondary antirabbit immunoglobulin $\mathrm{G}$ antibody at a dilution of 1:10000 (Abcam, Cambridge, MA, USA) was utilized to treat the membrane for $60 \mathrm{~min}$. ImageJ software (National Institutes of Health, USA) was utilized to visualize the band. Each test was repeated in triplicate.

\section{Immunohistochemistry}

PBS with 4\% paraformaldehyde was utilized to fix the tissue specimens, and embedded in paraffin. Xylenes were utilized to deparaffinize the sections, a graded ethanol series was utilized to rehydrate the sections and hematoxylin and eosin was utilized to stain the section. The histological score of each slide were evaluated and compared between the genotype groups.

\section{Statistical analysis}

SPSS 10.0 for Windows software package (SPSS Inc., Chicago, IL, USA) was utilized to carry out statistical analysis, all results were expressed as the means \pm standard deviation (SD). The values of $P<0.05$ were considered to be statistical significance.

\section{CONCLUSIONS}

Taken together, data from this study demonstrated, for the first time to our knowledge, that lncRNAs (GAS5) may be involved in the control of SMAD/TGF- $\beta 1$ -mediated renal fibrosis via targeting miR-21. Our findings provided evidence that rs145204276 may contribute to renal fibrosis via transcription alternation of the GAS5 promoter, thus possibly serving as a potential therapeutic target for renal fibrosis.

\section{CONFLICTS OF INTEREST}

None.

\section{REFERENCES}

1. Nath KA. Tubulointerstitial changes as a major determinant in the progression of renal damage. Am J Kidney Dis. 1992; 20:1-17.

2. Campanholle G, Ligresti G, Gharib SA, Duffield JS. Cellular mechanisms of tissue fibrosis. 3. Novel mechanisms of kidney fibrosis. Am J Physiol Cell Physiol. 2013; 304:C591-603. 
3. Eddy AA. Molecular basis of renal fibrosis. Pediatr Nephrol. 2000; 15:290-301.

4. Zhong X, Chung AC, Chen HY, Meng XM, Lan HY. SMAD3mediated upregulation of miR-21 promotes renal fibrosis. J Am Soc Nephrol. 2011; 22:1668-81.

5. Zhou Q, Chung AC, Huang XR, Dong Y, Yu X, Lan HY. Identification of novel long noncoding RNAs associated with TGF-beta/SMAD3-mediated renal inflammation and fibrosis by RNA sequencing. Am J Pathol. 2014; 184:40917.

6. Mattick JS. The genetic signatures of noncoding RNAs. PLoS Genet. 2009; 5:e1000459.

7. Wahlestedt C. Targeting long non-coding RNA to therapeutically upregulate gene expression. Nat Rev Drug Discov. 2013; 12:433-46.

8. Vettori S, Gay S, Distler O. Role of MicroRNAs in Fibrosis. Open Rheumatol J. 2012; 6:130-9.

9. Wang J, Gao Y, Ma M, Li M, Zou D, Yang J, Zhu Z, Zhao $\mathrm{X}$. Effect of miR-21 on renal fibrosis by regulating MMP9 and TIMP1 in kk-ay diabetic nephropathy mice. Cell Biochem Biophys. 2013; 67:537-46.

10. Chau BN, Xin C, Hartner J, Ren S, Castano AP, Linn G, Li J, Tran PT, Kaimal V, Huang X, Chang AN, Li S, Kalra A, et al. MicroRNA-21 promotes fibrosis of the kidney by silencing metabolic pathways. Sci Transl Med. 2012; 4: $121 \mathrm{ra} 18$

11. Zhong X, Chung AC, Chen HY, Dong Y, Meng XM, Li R, Yang W, Hou FF, Lan HY. miR-21 is a key therapeutic target for renal injury in a mouse model of type 2 diabetes. Diabetologia. 2013; 56:663-74.

12. McClelland AD, Herman-Edelstein M, Komers R, Jha JC, Winbanks CE, Hagiwara S, Gregorevic P, Kantharidis P, Cooper ME. miR-21 promotes renal fibrosis in diabetic nephropathy by targeting PTEN and SMAD7. Clin Sci (Lond). 2015; 129:237-49.

13. Schneider C, King RM, Philipson L. Genes specifically expressed at growth arrest of mammalian cells. Cell. 1988; 54:787-93.

14. Zhang Z, Zhu Z, Watabe K, Zhang X, Bai C, Xu M, Wu F, Mo YY. Negative regulation of lncRNA GAS5 by miR-21. Cell Death Differ. 2013; 20:1558-68.

15. Tao R, Hu S, Wang S, Zhou X, Zhang Q, Wang C, Zhao X, Zhou W, Zhang S, Li C, Zhao H, He Y, Zhu S. Association between indel polymorphism in the promoter region of lncRNA GAS5 and the risk of hepatocellular carcinoma. Carcinogenesis. 2015; 36:1136-43.

16. Loboda A, Sobczak M, Jozkowicz A, Dulak J. TGF-beta1/ SMADs and miR-21 in Renal Fibrosis and Inflammation. Mediators Inflamm. 2016; 2016:8319283.

17. Hu L, Ye H, Huang G, Luo F, Liu Y, Liu Y, Yang X, Shen J, Liu Q, Zhang J. Long noncoding RNA GAS5 suppresses the migration and invasion of hepatocellular carcinoma cells via miR-21. Tumour Biol. 2016; 37:2691-702.
18. Cao S, Liu W, Li F, Zhao W, Qin C. Decreased expression of lncRNA GAS5 predicts a poor prognosis in cervical cancer. Int J Clin Exp Pathol. 2014; 7:6776-83.

19. Kang L, Sun J, Wen X, Cui J, Wang G, Hoffman AR, Hu JF, Li W. Aberrant allele-switch imprinting of a novel IGF1R intragenic antisense non-coding RNA in breast cancers. Eur J Cancer. 2015; 51:260-70.

20. Wang J, Liu X, Wu H, Ni P, Gu Z, Qiao Y, Chen N, Sun F, Fan Q. CREB up-regulates long non-coding RNA, HULC expression through interaction with microRNA-372 in liver cancer. Nucleic Acids Res. 2010; 38:5366-83.

21. Song J, Ahn C, Chun CH, Jin EJ. A long non-coding RNA, GAS5, plays a critical role in the regulation of miR-21 during osteoarthritis. J Orthop Res. 2014; 32:1628-35.

22. Liu G, Friggeri A, Yang Y, Milosevic J, Ding Q, Thannickal VJ, Kaminski N, Abraham E. miR-21 mediates fibrogenic activation of pulmonary fibroblasts and lung fibrosis. J Exp Med. 2010; 207:1589-97.

23. Lan HY, Chung AC. TGF-beta/SMAD signaling in kidney disease. Semin Nephrol. 2012; 32:236-43.

24. Roberts AB, Tian F, Byfield SD, Stuelten C, Ooshima A, Saika S, Flanders KC. SMAD3 is key to TGF-betamediated epithelial-to-mesenchymal transition, fibrosis, tumor suppression and metastasis. Cytokine Growth Factor Rev. 2006; 17:19-27.

25. Qin W,Chung AC, Huang XR, Meng XM, Hui DS, Yu CM, Sung JJ, Lan HY. TGF-beta/SMAD3 signaling promotes renal fibrosis by inhibiting miR-29. J Am Soc Nephrol. 2011; 22:1462-74.

26. Roberts AB. Molecular and cell biology of TGF-beta. Miner Electrolyte Metab. 1998; 24:111-9.

27. Lan HY, Chung AC. Transforming growth factor-beta and SMADs. Contrib Nephrol. 2011; 170:75-82.

28. Davis BN, Hilyard AC, Lagna G, Hata A. SMAD proteins control DROSHA-mediated microRNA maturation. Nature. 2008; 454:56-61.

29. Cheng YH. Estimation of teaching-learning-based optimization primer design using regression analysis for different melting temperature calculations. IEEE Trans Nanobioscience. 2015; 14:3-12.

30. Garcia R, Nistal JF, Merino D, Price NL, FernándezHernando C, Beaumont J, González A, Hurlé MA, Villar AV. p-SMAD2/3 and DICER promote pre-miR-21 processing during pressure overload-associated myocardial remodeling. Biochim Biophys Acta. 2015; 1852:1520-30.

31. Chung AC, Huang XR, Zhou L, Heuchel R, Lai KN, Lan HY. Disruption of the SMAD7 gene promotes renal fibrosis and inflammation in unilateral ureteral obstruction (UUO) in mice. Nephrol Dial Transplant. 2009; 24:1443-54.

32. Inazaki K, Kanamaru Y, Kojima Y, Sueyoshi N, Okumura K, Kaneko K, Yamashiro Y, Ogawa H, Nakao A. SMAD3 deficiency attenuates renal fibrosis, inflammation, and apoptosis after unilateral ureteral obstruction. Kidney Int. 2004; 66:597-604. 